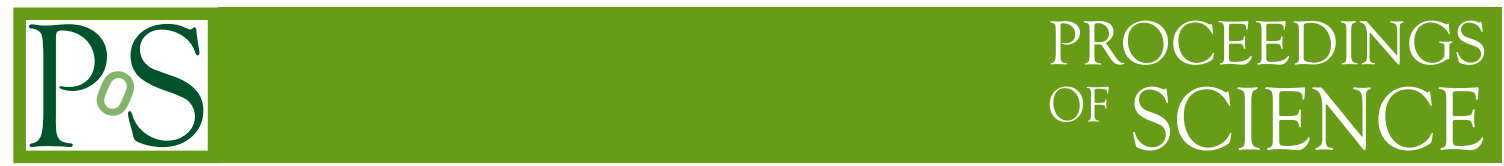

\title{
The Two Hemispheres Method for Multijet BSM Searches
}

\author{
Daniel Turgeman, ${ }^{a, *}$ Michael Pitt $^{b}$ and Ehud Duchovni ${ }^{a}$ \\ ${ }^{a}$ Weizmann Institute of Science \\ 234 Herzl st, Rehovot, Israel \\ ${ }^{b}$ CERN \\ CH 1211, Geneva 23, Switzerland \\ E-mail: Daniel.Turgeman@weizmann.ac.il
}

\begin{abstract}
A new method for identifying hints of possible beyond the standard model (BSM) signals produced at the Large Hadron Collider (LHC) with high jet multiplicity final states is proposed. In particular, the QCD background is estimated in a data driven way. Based on the simplified picture where QCD multijet events are created from a $2 \rightarrow 2$ process followed by cascade branching of the outcoming partons, the proposed "Two Hemisphere Method" (THm) divides events to two hemispheres and predicts the distribution of the number of jets in a predefined high multiplicity signal region. Validation of the above-mentioned assumption was performed using LO, NLO, and NNLO simulations, showing no effect of higher order calculations on the prediction accuracy.

The sensitivity of the method was examined on topologically distinct scenarios of BSM multijet signatures and was able to show comparable sensitivity to other methods used in previous analyses. Since the sources of the uncertainties in this new approach are very different from the current methods, the procedures complement one another.
\end{abstract}

The Eighth Annual Conference on Large Hadron Collider Physics-LHCP2020

25-30 May, 2020

online

${ }^{*}$ Speaker 


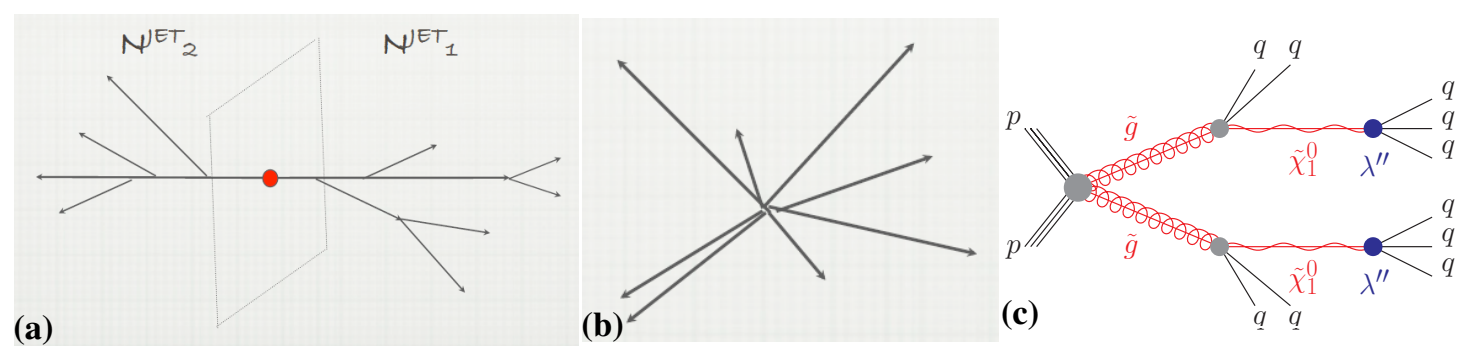

Figure 1: Illustrations of a multijet event produced by (a) two back-to-back out-coming partons each followed by a parton shower (b) micro black hole decay (c) RPV SUSY gluino cascade decay chain.

\section{Introduction}

Several BSM models (such as Micro-Black-Holes (mBH) or R-Parity Violation (RPV) Supersymmetry) predict the possible production at the LHC of events with a large number of outcoming high energy partons. These events will give rise to final states consisting of a high multiplicity of energetic jets, i.e. multi-jet events. The identification of this type of signal through the observation of an excess of multijet events is nontrivial due to the presence of large SM background originating from QCD processes. Background estimation via simulation is limited and therefore a data driven approach is favorable.

\section{Concept and Method}

As a first order approximation QCD events can be seen as beginning with a $2 \rightarrow 2$ process that gives rise to two back-to-back out-coming partons, followed by a parton shower (Fig. 1a). Since the showering is carried out for each parton independently it is claimed here that at first approximation the number of jets in each hemisphere are not correlated. This hypothesis was validated for higher orders using NLO and NNLO generators in [1].

On the other hand, multijet signatures originating from massive BSM states (Fig. 1b and 1c) are expected to show stronger correlation between the jet multiplicities of each hemisphere. It is this difference that is utilized by the THm to predict the QCD background and differentiate a possible signal from that background. The procedure is outlined as follows:

Divide events into 2 hemispheres (A and B) using the plane perpendicular to the transverse thrust axis. The number of jets in each hemisphere will be denoted $\mathrm{N}_{\text {Jets }}^{\mathrm{A}}$ and $\mathrm{N}_{\text {Jets }}^{\mathrm{B}}$ respectively. For each value of $\mathrm{N}_{\text {Jets, }}^{\mathrm{A}}$, obtain the distribution of $\mathrm{N}_{\text {Jets }}^{\mathrm{B}}$ (illustrated in Fig. 2).

Under the simplistic $2 \rightarrow 2$ assupmtion the showering initiated by each outcoming parton is independant of the other outcoming parton, therefore the normalized $\mathrm{N}_{\mathrm{Jets}}^{\mathrm{B}}$ distributions should be similar for any chosen $\mathrm{N}_{\text {Jets. }}^{\mathrm{A}}$. This is shown in Fig. 3 using a LO generator calculation integrated with a parton shower (Pythia) and was validated using NLO and NNLO generators in [1] .

The hypothetical High $\mathrm{N}_{\text {Jet }}$ signal is unlikely to give rise to highly asymmetric events with a large jet multiplicity in hemisphere $B$ and only one jet in hemisphere $A$. Therefore, the $\mathrm{N}_{\text {Jets }}^{\mathrm{B}}\left(\mathrm{N}_{\text {Jets }}^{\mathrm{A}}=1\right)$ distribution (black) should always be relatively signal free. $\mathrm{N}_{\text {Jets }}^{\mathrm{B}}\left(\mathrm{N}_{\text {Jets }}^{\mathrm{A}}=1\right)$ can thus be used to represent the $\mathrm{N}_{\text {Jets }}^{\mathrm{B}}$ distribution of pure QCD and serve as a QCD background estimation for those samples which might host signal events (i.e. $\mathrm{N}_{\text {Jets }}^{\mathrm{A}}=2,3,4$..). An excess of events with high jet 


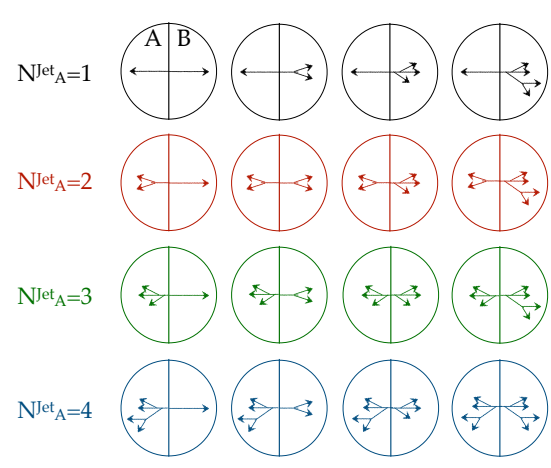

Figure 2: Representation for the distribution of the number of jets in hemisphere $\mathrm{B}\left(\mathrm{N}_{\text {Jets }}^{\mathrm{B}}\right.$, right hemisphere) for each value of $\mathrm{N}_{\text {Jets }}^{\mathrm{A}}$ (left hemisphere).

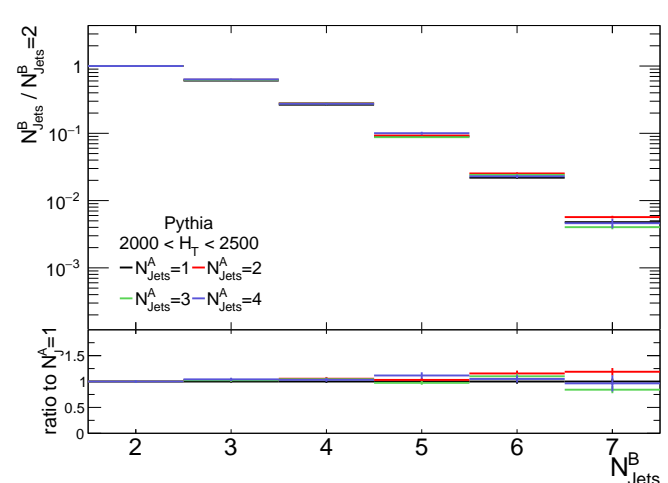

Figure 3: Normalized distributions of $\mathrm{N}_{\text {Jets }}^{\mathrm{B}}$ for each value of $\mathrm{N}_{\text {Jets. }}^{\mathrm{A}}$. As can be seen all distributions are in close agreement.

multiplicity may be considered as a possible indication for the presence of a signal (Fig. 4 bottom right).

\section{Simulation}

QCD events were generated and showered with PYTHIA8 [2] at $\sqrt{s}=13 \mathrm{TeV}$ using the Monash 2013 tune [3]. Jets were reconstructed using the anti-kt algorithm [4] implemented in the FastJet 3.2.1 package [5] with a radius parameter value of $R=0.4$. All jets were required to satisfy $\mathrm{p}_{\mathrm{T}}>50$ $\mathrm{GeV}$ and $|\eta|<2.8$. $\mathrm{mBH}$ signal samples were produced using a semi-classical approximation with the CHARYBDIS2 [6] event generator. The signal sample parameters include the number of extra dimensions $(n)$, the value of the diminished Planck mass $\left(M_{D}\right)$ and the threshold above which the semi-classical treatment of the microscopic $\mathrm{BH}$ is expected to be valid $\left(\mathrm{M}_{\mathrm{th}}\right)$. The production crosssection for each sample is determined by its parameters. Multijet RPV SUSY signal samples were produced using the MadGraph5_aMC@NLO [7] event generator, parton showered with PYTHIA 8. Cascade gluino decay samples were produced for values of $1000<m_{\tilde{g}}<2100 \mathrm{GeV}$ and neutralino mass $50 \mathrm{GeV}<m_{\tilde{\chi}_{1}^{0}}<1.65 \mathrm{TeV}$ (where always $m_{\tilde{\chi}_{1}^{0}}<m_{\tilde{g}}$ ).

\section{Results}

In order to test the sensitivity of the above outlined search procedure, simulated signals of two topologically distinct scenarios, $\mathrm{mBH}$ and RPV SUSY, were added to the simulated QCD sample. These simulated signal events revealed that the $\mathrm{mBH}$ samples have lower average jet multiplicty and larger variance thus giving rise to a non-negligible signal contamination in the supposedly signal free $\mathrm{N}_{\text {Jets }}^{\mathrm{A}}=1$ (Fig. 4 top left). Such signal contamination of the conjectured "signal-free" prediction deems that the THm sensitivity for these $\mathrm{mBH}$ samples is much reduced (Fig. 4 bottom left). The RPV SUSY signal samples were found to have higher average jet multiplicity and a smaller variance thus leading to negligible contamination in $\mathrm{N}_{\text {Jets }}^{\mathrm{A}}=1$ (Fig. 4 top right) and therefore provide a signal that can potentially be detected by the THm (Fig. 4 bottom right). Figure 5 shows the expected limit at 95\% CL using the Two Hemispheres method (blue) overlaid on the exclusion contour from the ATLAS RPV multijet analysis [8]. Exclusion contours given in the $\left(m_{\tilde{g}}, m_{\tilde{\chi}_{1}^{0}}\right)$ plane for the 

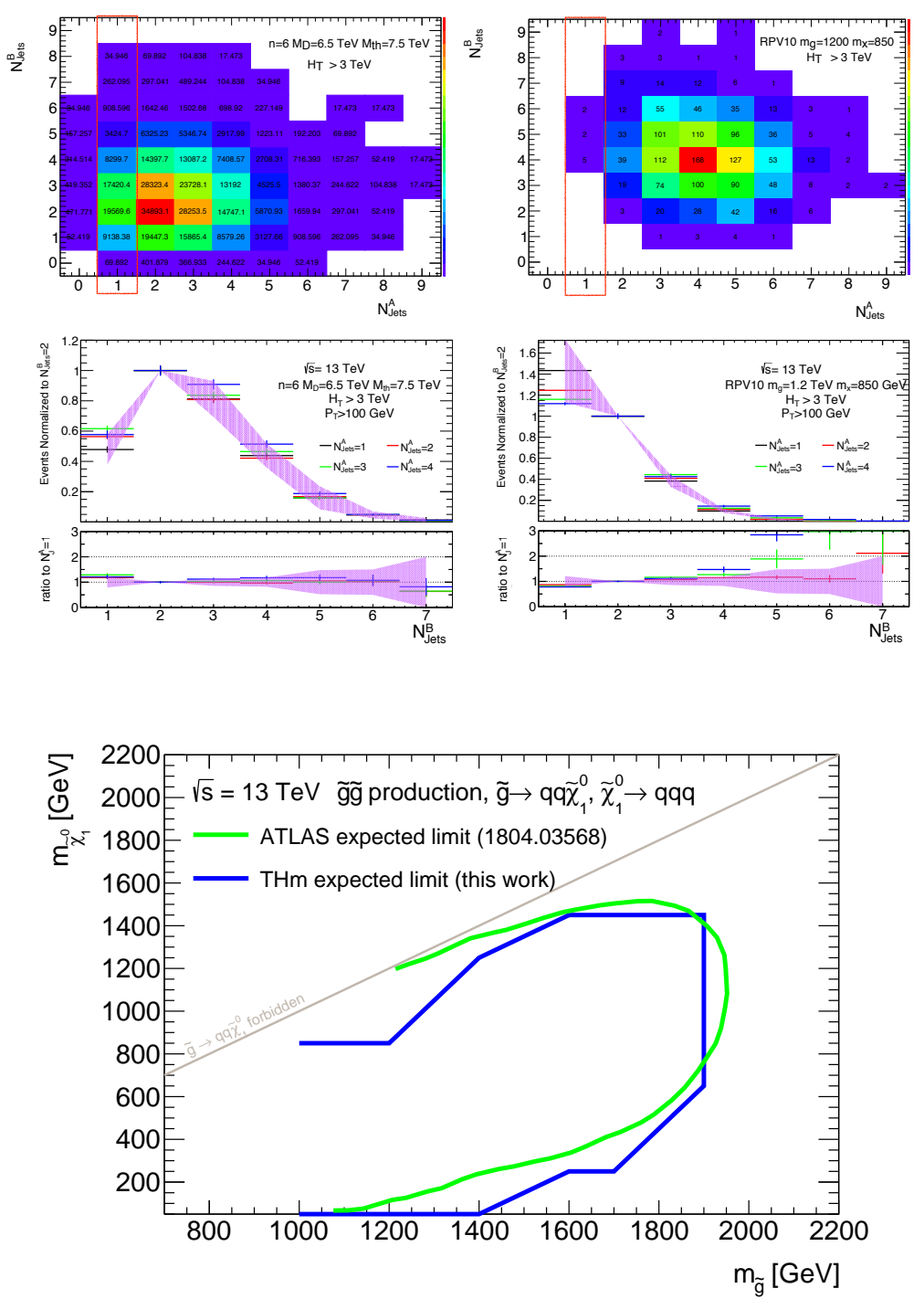

Figure 4: Top: $\mathrm{N}_{\text {Jets }}^{\mathrm{A}}$ vs. $\quad \mathrm{N}_{\text {Jets }}^{\mathrm{B}}$ of characteristic $\mathrm{mBH}$ sample (left) showing significant ( $\sim 20 \%$ of total sample) signal contamination in $\mathrm{N}_{\text {Jets }}^{\mathrm{A}}=1$ (red rectangle). RPV SUSY (right) showing negligible contamination. Bottom: Normalized $\mathrm{N}_{\text {Jets }}^{\mathrm{B}}$ distributions for SM bkg (Pythia) injected with signal. RPV SUSY (right) causes $\mathrm{N}_{\text {Jets }}^{\mathrm{A}}=3,4$ (green, blue) to deviate significantly from the $\mathrm{N}_{\text {Jets }}^{\mathrm{A}}=1$, thus exposing signal as desired. $\mathrm{mBH}$ (left) suffers from significant signal contamination in $\mathrm{N}_{\text {Jets }}^{\mathrm{A}}=1$ thus greatly reducing sensitivity

Figure 5: Expected limits (95\% CL) for the RPV SUSY gluino cascade decay model using the THm (blue) compared to those achieved by the ATLAS RPV multijet analysis (green).

gluino cascade decay model. The results are comparable to those achieved in the ATLAS paper (interpolation between the points of the signal parameter grid was not perfomed for the THm). The THm is new and further optimization may improve sensitivity. Yet, even with similar sensitivity the two analyses, being so different, complement each other. Should a signal be detected in one analysis the other can be used to confirm or refute its existence.

\section{References}

[1] D. Turgeman, M. Pitt, I. Roth and E. Duchovni, On the Modelling of Energetic Multi-jet QCD Events, (to appear in EPJC), 1912.01254.

[2] T. Sjostrand, S. Ask, J. R. Christiansen, R. Corke, N. Desai, P. Ilten et al., An Introduction to PYTHIA 8.2, Comput. Phys. Commun. 191 (2015) 159-177, [1410.3012]. 
[3] P. Skands, S. Carrazza and J. Rojo, Tuning PYTHIA 8.1: the Monash 2013 Tune, Eur. Phys. J. C74 (2014) 3024, [1404.5630].

[4] M. Cacciari, G. P. Salam and G. Soyez, The anti- $k_{t}$ jet clustering algorithm, JHEP 04 (2008) 063, [0802.1189].

[5] M. Cacciari, G. P. Salam and G. Soyez, FastJet User Manual, Eur. Phys. J. C72 (2012) 1896, [1111.6097].

[6] J. A. Frost, J. R. Gaunt, M. O. Sampaio, M. Casals, S. R. Dolan et al., Phenomenology of Production and Decay of Spinning Extra-Dimensional Black Holes at Hadron Colliders, JHEP 0910 (2009) 014, [0904 . 0979].

[7] J. Alwall, R. Frederix, S. Frixione, V. Hirschi, F. Maltoni, O. Mattelaer et al., The automated computation of tree-level and next-to-leading order differential cross sections, and their matching to parton shower simulations, JHEP 07 (2014) 079, [1405.0301].

[8] ATLAS collaboration, M. Aaboud et al., Search for R-parity-violating supersymmetric particles in multi-jet final states produced in p-p collisions at $\sqrt{s}=13 \mathrm{TeV}$ using the ATLAS detector at the LHC, Phys. Lett. B785 (2018) 136-158, [1804 . 03568]. 